\title{
Time Evolution of the Spatial Structure of the Radial Electric Field in the Tohoku University Heliac
}

\author{
Hiroshi AOYAMA, Sumio KITAJIMA, Mamiko SASAO, Atsushi OKAMOTO, \\ Takashi KOBUCHI, Yutaka TANAKA, Hiroyasu UTOH, Hajime UMETSU, \\ Keiichi ISHII, and Hiromi TAKAHASHI ${ }^{1)}$ \\ Department of Quantum Science and Energy Engineering, Tohoku University, Sendai 980-8579, Japan \\ 1) National Institute for Fusion Science, 322-6 Oroshi-cho, Toki 509-5292, Japan
}

(Received 16 November 2007 / Accepted 17 March 2008)

\begin{abstract}
Radial electric fields are closely related to the confinement of plasma. Electrode biasing experiments were conducted to actively control the radial electric field by ramping up/down the electrode current in the Tohoku University Heliac (TU-Heliac). An emissive probe array consisting of three filaments was designed as a new type of measurement equipment. Using this probe, time evolutions of the spatial structure of the radial electric field were measured in the biasing experiments. The radial electric field tended to extend from the inner region to the outer region, while ramping up the electrode current. In the plasma inner region, the radial electric field was maintained for a longer time than in the outer region, while ramping down the electrode current. The radial electric field, poloidal flow of plasma, and fluctuation level of the ion saturation current changed appreciably in the period when the electrode voltage showed negative resistance against the electrode current. Therefore, the period when the plasma shows negative resistance agreed with the transition region to the improved confinement mode.
\end{abstract}

(c) 2008 The Japan Society of Plasma Science and Nuclear Fusion Research

Keywords: radial electric field, emissive probe, electrode biasing experiment, LH transition, fluctuation

DOI: $10.1585 /$ pfr.3.S1026

\section{Introduction}

The neoclassical theory predicts that the ion viscosity has local maxima against the poloidal rotation velocity [1]. Rapid increases in the poloidal rotation occur when the driving force in the poloidal direction exceeds a critical value, at which the plasma is considered to transit into the improved confinement mode. One of the characteristics of the transition into the improved mode is a sudden growth of the radial electric field [2]. Therefore, it is important to investigate the time evolution of the spatial structure of the radial electric field for understanding the detailed transition mechanism. The radial electric field can be controlled actively by electrode biasing. In fact, electrode biasing experiments have been conducted using various devices [3,4]. In addition, the shear of the radial electric field plays an important role in the improvement of the plasma confinement by suppressing the fluctuation level $[5,6]$.

In the TU-Heliac, electrode biasing experiments using a hot cathode composed of $\mathrm{LaB}_{6}$ have been conducted to control the transition into the improved mode $[7,8]$. We performed forward/reverse transition experiments by ramping up/down the electrode current. In these experiments, the negative plasma resistance and the hysteresis between the radial electric field and the stored energy were observed in the transition region [9]. The nonlinear change

author's e-mail: hiroshi.aoyama@ppl2.qse.tohoku.ac.jp in the electrode voltage against the electrode current is one of the characteristics of the transition. To understand the behavior of the radial electric field in the transition region, it is important to measure the time evolution of the spatial structure of the radial electric field around the period when the electrode voltage shows nonlinearity against the electrode current. For this purpose, we developed an emissive probe array to define the radial electric field. Using this probe, we measured the distributions of the radial electric field, and compared them with the results of a Mach probe and the fluctuation level of the ion saturation current.

\section{Experimental Setup}

The TU-Heliac is a small heliac device with three sets of magnetic field coils. The top view of the TU-Heliac is shown in Fig. 1. The size parameters of this device are as follows: the major radius $R_{0}=0.48 \mathrm{~m}$, and the average plasma radius $a \sim 0.07 \mathrm{~m}$. In the electrode biasing experiments, the target plasma was He plasma produced by alternative ohmic heating with $f=18.8 \mathrm{kHz}, P \sim 35 \mathrm{~kW}$, and the discharge time was $10 \mathrm{~ms}$. The electron temperature $T_{\mathrm{e}}$, electron density $n_{\mathrm{e}}$, floating potential $V_{\mathrm{f}}$, and their fluctuation levels were measured using the triple probe placed at a toroidal angle $\phi$ of $0^{\circ}$.

The hot cathode composed of $\mathrm{LaB}_{6}$ was used as an electrode in the electrode biasing experiments. The hot 


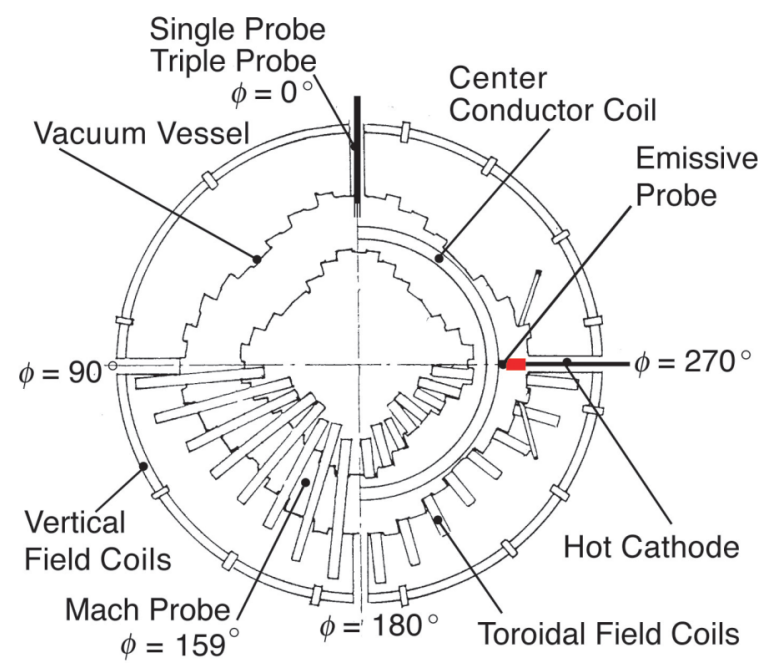

Fig. 1 Top view of TU-Heliac.

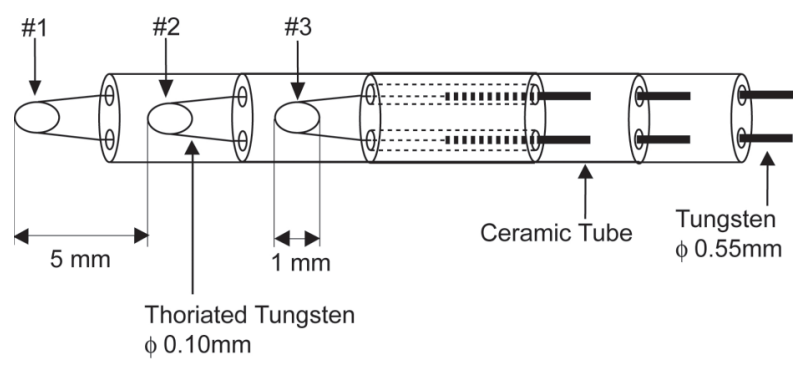

Fig. 2 Schematic of the emissive probe array

cathode was cylindrical in shape, $17 \mathrm{~mm}$ in length, and $10 \mathrm{~mm}$ in diameter. This cathode was inserted into the plasma horizontally at $\phi=270^{\circ}$. The electrode was negatively biased against the vacuum vessel by a current control power supply.

The plasma poloidal flow is related closely to the radial electric field $E_{\mathrm{r}}$. The Mach probe was used to compare $E_{\mathrm{r}}$ with the plasma poloidal flow. It was inserted vertically into the plasma from the upper port at $\phi=159^{\circ}$.

We designed the emissive probe array shown in Fig. 2, which was composed of three filaments made of $1 \%$ thoriated tungsten (Th-W) with a diameter of $0.1 \mathrm{~mm}$. Th-W is a very useful material for emissions of electrons at low temperatures compared with pure tungsten. The intervals of each filament were $5 \mathrm{~mm}$. The Th-W wires were $30 \mathrm{~mm}$ in length, and were bent into a circle with a diameter of $1 \mathrm{~mm}$. Both ends of the wires were connected to the tungsten wires with a diameter of $0.55 \mathrm{~mm}$ in a doubly drilled ceramic tube. This probe was inserted vertically into the plasma from the upper port at $\phi=270^{\circ}$. All filaments were set facing toward the magnetic axis. This setting was adopted to avoid the intersection of the plasma flow and the magnetic field line. When the filaments are heated, they start to emit electrons and the floating potential measured

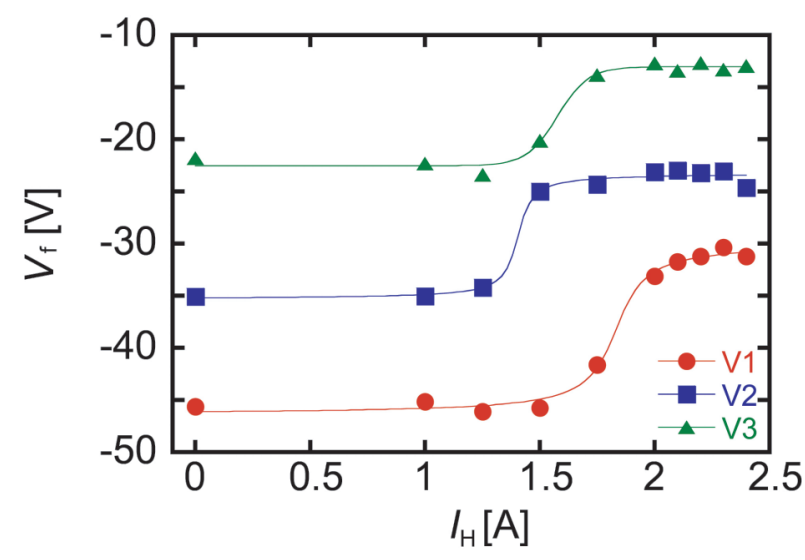

Fig. 3 Relations between heating current $I_{\mathrm{H}}$ and the floating potential $V_{\mathrm{f}}$ measured by emissive probe array. \#1- 3 are the filament number (see Fig. 2).

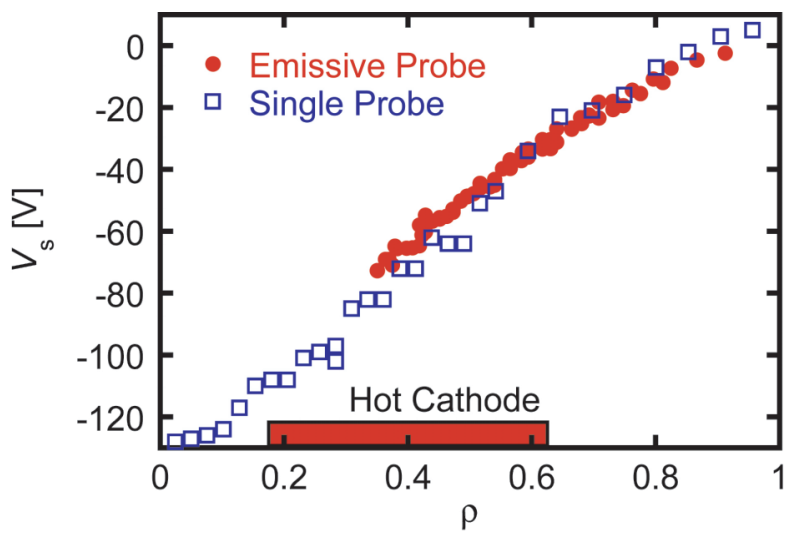

Fig. 4 Radial profile of the plasma space potential measured by single probe and emissive probe. Red circles and blue rectangles indicate the $V_{\mathrm{s}}$ measured by the emissive probe array and the single probe, respectively.

by the emissive probe shifts to the plasma space potential $V_{\mathrm{s}}$. The advantage of using an emissive probe over other Langmuir probe methods is that direct measurement of $V_{\mathrm{s}}$ is possible. In this experiment, the electron density was $n_{\mathrm{e}} \sim 10^{12} \mathrm{~cm}^{-3}$, and the filaments were heated to about $2000 \mathrm{~K}$ or less by a heating current of about 2 to $2.5 \mathrm{~A}$. Figure 3 shows the relation between the heating current $I_{\mathrm{H}}$ and the floating potential $V_{\mathrm{f}}$ measured using the emissive probe array. The floating potential measured using the emissive probe array was almost saturated in the heating current range from 2 to $2.5 \mathrm{~A}$. This probe can measure $V_{\mathrm{s}}$ at three points at the same time, allowing simultaneous measurement of $E_{\mathrm{r}}$ at two points.

The radial profile of $V_{\mathrm{s}}$ measured using a single probe (at toroidal angle $\phi=0^{\circ}$ ) and the emissive probe array (at toroidal angle $\phi=270^{\circ}$ ) is shown in Fig. 4. One of the pins of the triple probe was used for the single probe. These $V_{\mathrm{s}}$ values were measured in the period when the plasma confinement mode was the improved mode. The $V_{\mathrm{s}}$ values 
measured using the emissive probe array include the results of three emissive probes. As can be seen in Fig. 4, the results of the emissive probe array agreed well with those of the single probe.

\section{Experimental Results}

Figure 5 shows the typical time evolution of the electrode current $I_{\mathrm{E}}$ and the electrode voltage $V_{\mathrm{E}}$. $I_{\mathrm{E}}$ was controlled such that (a) it was ramped up to -4 A for $6 \mathrm{~ms}$ starting from $4 \mathrm{~ms}$, or (b) it was maintained at $-4 \mathrm{~A}$ at first and then ramped down to $0 \mathrm{~A}$ for $6 \mathrm{~ms}$. In these experiments, the hot cathode was located at $\rho=0.18-0.62$, where $\rho$ is the normalized minor radius defined by $\rho=\langle r\rangle / a$, and $\langle r\rangle$ is the average radius of the flux surface. $V_{\mathrm{E}}$ showed nonlinearity against $I_{\mathrm{E}}$ in the period surrounded by broken lines.

The radial profiles of $V_{\mathrm{s}}$ measured by the \#1 filament in the emissive probe array are shown in Fig. 6. Figure 6 (a) shows the results of the discharges of the results of $I_{\mathrm{E}}$ ramped up, and (b) shows those of $I_{\mathrm{E}}$ ramped down. Each profile was obtained from 18 discharges. The broken lines in Fig. 6 correspond to those shown in Fig. 5, and the solid line indicates the start time of the ramping up/down. As can be seen in Fig. 6 (a), $V_{\mathrm{s}}$ became deeper in the negative direction, starting from the plasma inner region when $I_{\mathrm{E}}$ was ramped up. In Fig. 6(b), when $I_{\mathrm{E}}$ was ramped down, $V_{\mathrm{s}}$ became shallow from the plasma outer region. Similar results were also obtained with the other two filaments \#2 and \#3. $V_{\mathrm{s}}$ near the magnetic axis $(\rho<0.35)$ could not be measured using the emissive probe array, because the probe scanned the plasma along the chord that was off-

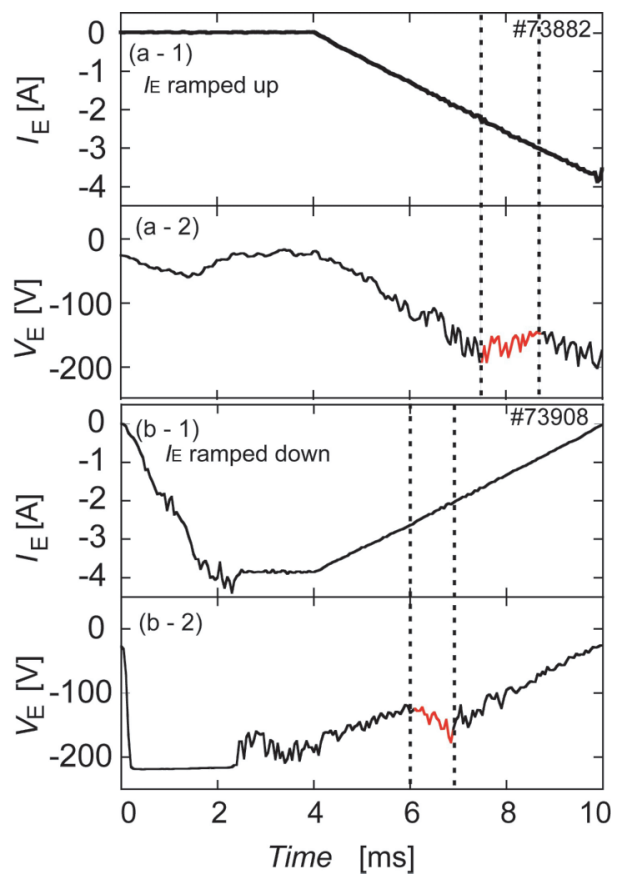

Fig. 5 Typical time evolution of $V_{\mathrm{E}}$ and $I_{\mathrm{E}}$ : (a) discharge with $I_{\mathrm{E}}$ ramped up; (b) discharge with $I_{\mathrm{E}}$ ramped down. center to the magnetic axis.

Figure 7 shows the time evolution of the spatial structure of (a) $E_{\mathrm{r}}$, (b) the current ratio of the Mach probe, and (c) the fluctuation level of the ion saturation current in the discharges of $I_{\mathrm{E}}$ ramped up, corresponding to Fig. 5 (a). $E_{\mathrm{r}}$ was calculated from the differential of $V_{\mathrm{s}}$, measured with filaments \#1 and \#3. In Fig. 7 (b), $I_{\text {up }}$ and $I_{\text {down }}$ indicate the ion saturation currents that flowed into the pins at the upstream and downstream sides, respectively. The current ratio of the Mach probe is considered to show plasma flow, which is closely related to $E_{\mathrm{r}}$. Moreover, the suppression of the fluctuation level is also related to $E_{\mathrm{r}}$ and its shear. In this experiment, the fluctuation level was an exponent of the integral of the normalized power spectrum from 20 to $100 \mathrm{kHz}$, as shown in the following equation.

$$
\log \sum\left(C^{2}(\omega) / I_{\mathrm{is}}\right)
$$

Here, $C(\omega)$ is the power spectrum obtained from FFT, and $\omega$ is the frequency of the fluctuations. As can be seen in Fig. 7 (a), the strong $E_{\mathrm{r}}$ arose in the plasma inner region, and diffused in the plasma outer region. Figure 7 (b) indicates poloidal plasma flow in the electron diamagnetic direction, which is the same direction as $\boldsymbol{E} \times \boldsymbol{B}$ poloidal flow. The high-speed flow spread from inside to outside. Figure 7 (c) shows that the fluctuation level was suppressed after the second broken line $(t>8.7 \mathrm{~ms})$, especially in the inner region $(\rho<0.6)$. In the region of $\rho<0.7$ and $t>8 \mathrm{~ms}$, the region where the strong $E_{\mathrm{r}}$ was formed corresponded to the high current ratio region of the Mach probe and the low-fluctuation region. In the period surrounded by the broken line in Fig. 7, $E_{\mathrm{r}}$ and the current ratio were signif-

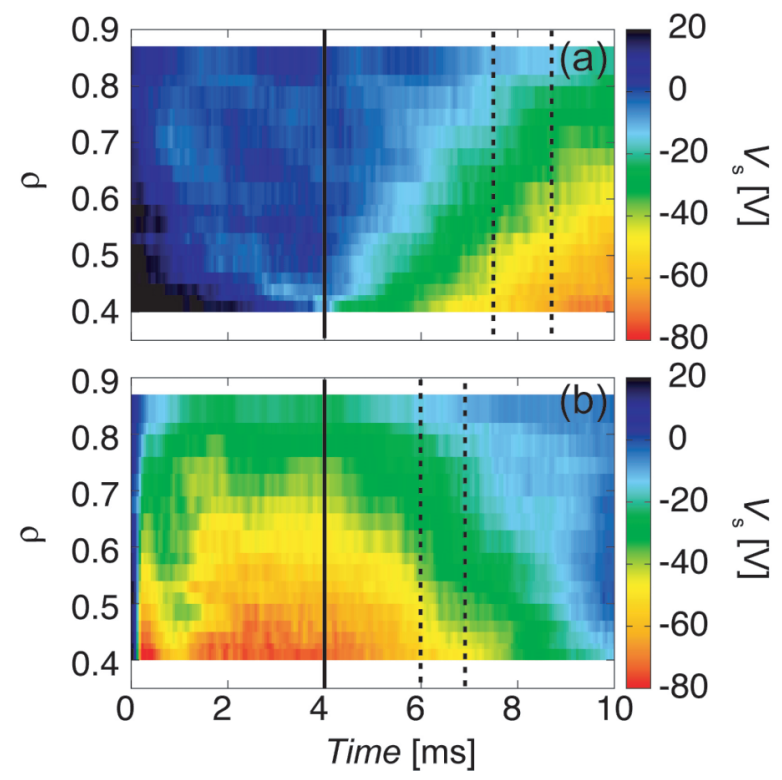

Fig. 6 Plasma space potential $V_{\mathrm{s}}$ measured with the emissive probe array in the discharge (a) $I_{\mathrm{E}}$ ramped up and (b) $I_{\mathrm{E}}$ ramped down. 


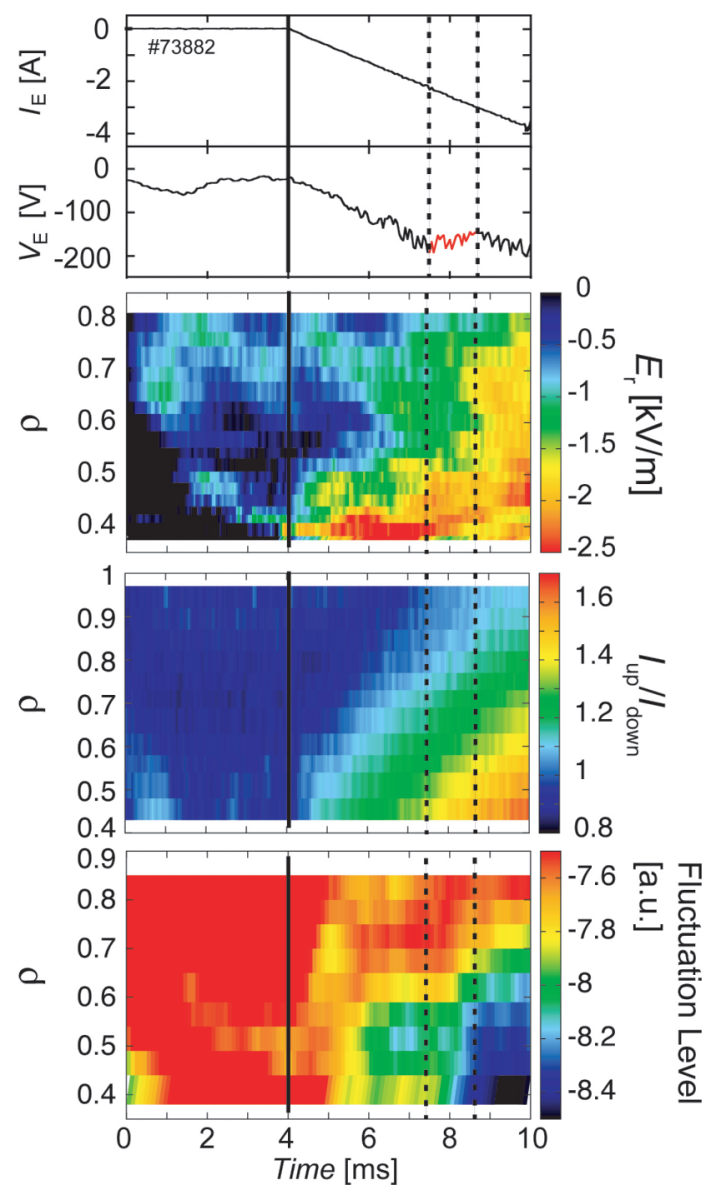

Fig. 7 The time evolution of the spatial structure of (a) the $E_{\mathrm{r}}$, (b) the current ratio of the Mach probe, and (c) the fluctuation level of the ion saturation current. All show the data in the discharges with $I_{\mathrm{E}}$ ramped up. The solid line indicates the start of ramping up. Broken lines are indicators of the nonlinear period.

icantly increased. In particular, in the region of $\rho>0.5$, $E_{\mathrm{r}}$ increased at the same time. In addition, after the second broken line, both were saturated at higher values. On the contrary, the fluctuation level changed gradually in this period and was suppressed remarkably after this period.

The results with the discharges of $I_{\mathrm{E}}$ ramped down are shown in Fig. 8. In the plasma inner region, a strong $E_{\mathrm{r}}$ was maintained for a longer time as compared to the outer region. The plasma flow gradually weakened from the outer region of the plasma. The fluctuation level was compressed to a low level during the period $2<t<6 \mathrm{~ms}$, corresponding to that with strong $E_{\mathrm{r}}$ and the large current ratio shown in Fig. 8 (a) and (b). These observations indicate that $E_{\mathrm{r}}$ suppressed the fluctuation level. In the period surrounded by the broken lines, $E_{\mathrm{r}}$ decreased appreciably, and the fluctuation level showed the maximum value in the outer region of the plasma. Although the current ratio did not change markedly in the outer region, a large change was seen in the inner region $(\rho<0.6)$. After the second broken line $(t>8 \mathrm{~ms}), E_{\mathrm{r}}$ became strong again and the fluctuation level decreased. It was suggested that $E_{\mathrm{r}}$ was
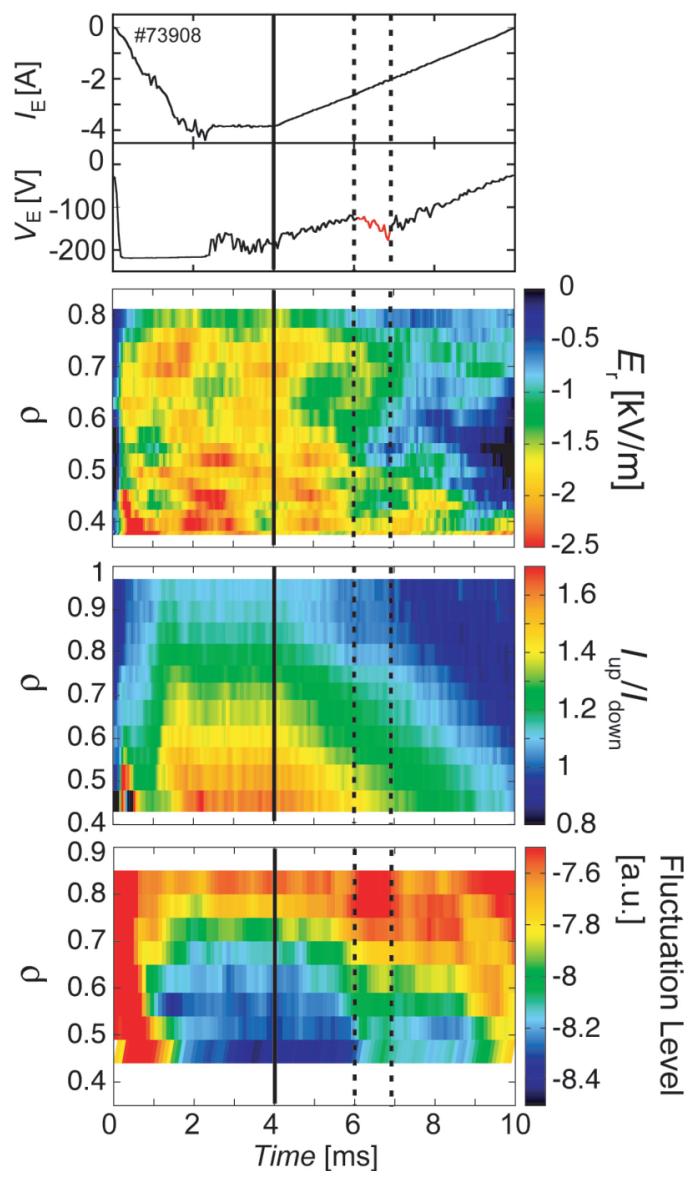

Fig. 8 The time evolution of the spatial structure of (a) the $E_{\mathrm{r}}$, (b) current ratio of the Mach probe, and (c) the fluctuation level of the ion saturation current. All show the data for the discharge with $I_{\mathrm{E}}$ ramped down. The solid line indicates the start of ramping up. Broken lines are indicators of the nonlinear period.

related to the suppression of the fluctuation.

In both the discharges with $I_{\mathrm{E}}$ ramped up and down (shown in Fig. 7 and Fig. 8) in the period surrounded by broken lines, a characteristic behavior of $E_{\mathrm{r}}$ was observed. In Fig. 7, in almost the entire region, a very small change in $E_{\mathrm{r}}$ was observed. In Fig. 8, in the region of $0.45<\rho<0.6$, $E_{\mathrm{r}}$ decreased and then rose again after this period. These changes in $E_{\mathrm{r}}$ did not correspond to the results of the Mach probe (Figs. 7 (b) and 8 (b)). The results of the Mach probe had simpler structures than those of the emissive probe array in the entire region. However, the observation that $E_{\mathrm{r}}$ decreased in the period surrounded by broken lines in Fig. 8 was consistent with the observation that the fluctuation level had the local maximum value in this period. This indicates that the emissive probe array can provide more detailed measurements than the Mach probe. These results show that the plasma had interesting characteristics in the period when the bias electrode voltage shows nonlinearity against the bias electrode current.

The strong $E_{\mathrm{r}}$, the high-speed flow and suppression of the fluctuation were observed at $t>8.7 \mathrm{~ms}$ in Fig. 7 and 
$t<6 \mathrm{~ms}$ in Fig. 8. These results suggested the characteristics of the improved mode. Therefore, the plasma nonlinearity region surrounded by the broken lines was the region of transition to the improved mode.

\section{Summary}

It was demonstrated that the emissive probe array had sufficient capability for measuring the time evolution of the spatial structure of the plasma space potential in electrode bias experiments in the TU-Heliac. The spatial profile of the radial electric field from the results of the plasma space potential was estimated. It was confirmed that the strong radial electric field spread from the inner region to the outer region in the discharge of $I_{\mathrm{E}}$ ramped up, and was maintained for a longer time in the inner region in the discharge of $I_{\mathrm{E}}$ ramped down. The characteristic region of the spatial and time profile of the radial electric field corresponded to those of the current ratio of the Mach probe and the fluctuation level of the ion saturation current, although the radial electric field showed weak correspondence to the plasma poloidal flow in the outer region of the plasma.
$E_{\mathrm{r}}$, the flow, and the fluctuation level changed appreciably in the period when the bias electrode voltage showed nonlinearity against the bias electrode current. Therefore, the region of plasma nonlinearity was the transition region to the improved confinement mode.

\section{Acknowledgment}

This study was supported by a Grant-in-Aid from the Ministry of Education, Science and Culture of Japan (No. 17360439).

[1] K.C. Shaing, Phys. Rev. Lett. 76, 4364 (1996).

[2] K.H. Burrell et al., Plasma Pys. Control. Fusion 31, 1649 (1989).

[3] R.J. Taylor et al., Phys. Rev. Lett. 63, 2365 (1989).

[4] E.Y. Wang et al., Nucl. Fusion 35, 467 (1995).

[5] H. Bigrali et al., Phys. Fluids B 2, 1 (1990).

[6] R.J. Groebner et al., Phys. Rev. Lett. 64, 3015 (1990).

[7] S. Kitajima et al., Nucl. Fusion 46, 200 (2006).

[8] S. Inagaki et al., Japan. J. Appl. Phys. 363697 (1997).

[9] H. Takahashi et al., Plasma Phys. Control. Fusion 48, 39 (2006). 